\title{
Variable universe adaptive fuzzy sliding mode projective synchronization of hyperjerk system based on disturbance observer
}

\author{
Baojie Zhang ${ }^{1,2}$ - Yuming Feng ${ }^{1} \cdot$ Hongyan $\mathrm{Yu}^{1} \cdot$ Xianxiu Zhang $^{2}$
}

Received: 26 June 2021 / Accepted: 3 December 2021

Published online: 20 December 2021

(c) The Author(s) 2021 OPEN

\begin{abstract}
In this paper, a sliding mode projective synchronization strategy based on disturbance observer and fuzzy system is presented to implement projective synchronization of hyperjerk system with low time-varying disturbance and white noise. Theoretical analysis and numerical calculation show that the disturbance observer can approach the low time-varying disturbance very well. The application of disturbance observer reduces the chattering of the controller. Variable universe adaptive fuzzy control (VUAFC) method is utilized to further reduce the chattering phenomenon. The simulation results demonstrate the effectiveness of the proposed controller.
\end{abstract}

Keywords Hyperjerk system · Sliding mode control · Disturbance observer · Low time-varying · Variable universe adaptive fuzzy control

\section{Introduction}

A hyperjerk system [1] is a dynamical system described by an $n$th ordinary differential equation where $n$ is 4 or up to, i.e.

$\frac{\mathrm{d}^{n} x}{\mathrm{~d} t^{n}}=J\left(\frac{\mathrm{d}^{n-1} x}{\mathrm{~d} t^{n-1}}, \ldots, \frac{\mathrm{d} x}{\mathrm{~d} t}, x\right) \quad(n \geqslant 4)$.

where $x$ denotes displacement, $\frac{\mathrm{d} x}{\mathrm{~d} t}$ denotes velocity, $\frac{\mathrm{d}^{2} x}{\mathrm{~d} t^{2}}$ is acceleration, $\frac{\mathrm{d}^{3} x}{\mathrm{~d} t^{3}}$ is jerk, and $\frac{\mathrm{d}^{4} \mathrm{x} t}{\mathrm{~d} t^{4}}, \ldots, \frac{\mathrm{d}^{n} x}{\mathrm{~d} t^{n}}$ are hyperjerks. When $n=3$, system (1) degenerates into a jerk system. The main concerns of the researchers are the chaotic or hyperchaotic performance, control and synchronization of jerk system or hyperjerk system with such simple structures [2-11].

As a synchronization strategy, the concept of projective synchronization of chaotic (or hyperchaotic) system was first proposed by Mainieri and Rehacek [12], where the introduction of a scaling factor extended the range of synchronization. When projective synchronization is realized, the state outputs of the drive system and the response system are not only phase locked, but also the amplitude of the corresponding state evolves according to a fixed proportional relationship. Because projective synchronization can be applied to chaotic secure communication, binary number can be extended to base $m$ number to achieve faster transmission, so many scholars have done in-depth research on projective synchronization [13-16].

For the inevitable external disturbance and uncertainty of control system, many control method is used, e.g., adaptive control $[7,11,17]$, backstepping control $[7,17,18]$, interval observer method [19, 20], sliding mode control [21-23]. A single control method cannot achieve the required precision of control, most of the control in the literature is the

Baojie Zhang, baojiezh@sanxiau.edu.cn; Yuming Feng, yumingfeng25928@qq.com; Hongyan Yu, yuhongyan@sanxiau.edu.cn; Xianxiu Zhang, 294312081@qq.com | ${ }^{1}$ College of Computer Science and Engineering, Chongqing Three Gorges University, Wanzhou 404000, Chongqing, People's Republic of China. ${ }^{2}$ Key Laboratory of Intelligent Information Processing and Control of Chongqing Municipal Institutions of Higher Education, Chongqing Three Gorges University, Wanzhou 404000, Chongqing, People's Republic of China. 
combination of several control methods. To address the problem of attitude adjustment for a quad-rotor unmanned aerial vehicle, Song et al. [17] apply the adaptive control and back-stepping control. One of the characteristics of sliding mode control is to overcome the uncertainty and disturbance of the system. Gao et al. [24] proposed the exponential reaching law method to design a sliding mode controller, which can not only accelerate the approach speed but also provide a measure for the reduction of chattering to some extent $[25,26]$. For the purpose of reaching the stable state of the control system in limited time, terminal sliding mode control is proposed by Zak [27]. Many researchers have applied the terminal sliding control to various control systems. Fei et al. [21, 23] designed the fractional-order sliding mode controller based on neural network.

In [28], Kawamura et al. proposed disturbance observer to compensate the uncertainty and disturbance of the servo motor in the controlling system. Some literature is posted on this method [29-31]. Considering the observer error and some other disturbance which could not compensate with observer, such as white noise, fuzzy system is a great idea [32] to smooth the discontinuity of the sliding mode control. Usually the control precision in a fuzzy system can be enhanced by increasing the amount of fuzzy rules. This can lead to a problem of a great deal of computations and even an "rules explosion." Variable universe adaptive fuzzy control method (VUAFC) introduced by Li $[33,34]$ can avoid the problem of "rules explosion" and improve the control precision by on-line contraction-expansion of variable universes.

Inspired by the above control methods, in this paper, a variable universe adaptive fuzzy sliding mode projective Synchronization (VUAFSMPS) based on disturbance observer is proposed for hyperjerk system. A sliding mode surface with nonlinear term is proposed. We quantitatively analyze the effect of the disturbance observer proposed in [28] and apply it to compensate the low time-varying disturbance of hyperjerk system. Variable universe adaptive fuzzy control method is used to smooth the switching term.

The organization of this work is as follows. In Sect. 2, problem description is given. In Sect. 3, variable universe adaptive fuzzy sliding mode projective synchronization is introduced in detail. In particular, we use linear system theory to analyze the observer error of disturbance observer. Simulation results given in Sect. 4. Finally, the conclusion is drawn.

\section{Problem description}

Hyperjerk system has a very simple structure. It is quite interesting because it presents a complex dynamic. In the literature, there are some hyperjerk systems presenting chaotic (hyperchaotic) phenomena. Here we consider the projective synchronization.

SN Applied Sciences
The drive system is depicted as

$$
\left\{\begin{array}{l}
\dot{x}_{1}=y_{1}, \\
\dot{y}_{1}=z_{1}, \\
\dot{z}_{1}=w_{1}, \\
\dot{w}_{1}=f_{d}\left(x_{1}, y_{1}, z_{1}, w_{1}\right)+d_{1}(t)+n_{1}(t)
\end{array}\right.
$$

where $x_{1}, y_{1}, z_{1}, w_{1}$ are state variables, $f_{d}$ is continuous function, $d_{1}(t)$ is an unknown low time-varying external disturbance, $\left|d_{1}(t)\right| \leqslant D_{1}, D_{1}$ is a positive constant, $n_{1}(t)$ is white noise, $n_{1}(t) \leqslant N_{1}$.

The response system is described as

$$
\left\{\begin{array}{l}
\dot{x}_{2}=y_{2} \\
\dot{y}_{2}=z_{2} \\
\dot{z}_{2}=w_{2} \\
\dot{w}_{2}=f_{r}\left(x_{2}, y_{2}, z_{2}, w_{2}\right)+d_{2}(t)+n_{2}(t)+u
\end{array}\right.
$$

where $x_{2}, y_{2}, z_{2}, w_{2}$ are state variables, $f_{r}$ is continuous function, $d_{2}(t)$ is an unknown low time-varying external disturbance, $\left|d_{2}(t)\right| \leqslant D_{2}, D_{2}$ is a positive constant, $n_{2}(t) \leqslant N_{2}, u$ is the controller to be designed.

Suppose the scaling factor is $\alpha$. The errors are given by

$$
\left\{\begin{array}{l}
e_{x}=x_{2}-\alpha x_{1} \\
e_{y}=y_{2}-\alpha y_{1} \\
e_{z}=z_{2}-\alpha z_{1} \\
e_{w}=w_{2}-\alpha w_{1}
\end{array}\right.
$$

Differentiating (4), the error dynamic system is

$$
\left\{\begin{array}{l}
\dot{e}_{x}=e_{y} \\
\dot{e}_{y}=e_{z^{\prime}} \\
\dot{e}_{z}=e_{w} \\
\dot{e}_{w}=f_{r}-\alpha f_{d}+\left(d_{2}(t)+n_{2}(t)\right)-\alpha\left(d_{1}(t)+n_{1}(t)\right)+u
\end{array}\right.
$$

Our goal is designing suitable input $u$ such that system (3) synchronizes (2) up to the scaling factor $\alpha$, i.e., system (5) converges to zero, especially, $\boldsymbol{e}=\left(e_{x}, e_{y}, e_{z}, e_{w}\right) \rightarrow 0$ as $t \rightarrow \infty$.

\section{Design of fuzzy sliding mode projective synchronization based on disturbance observer}

The sliding mode surface with nonlinear term is proposed in Sect. 3.1. The observer of slow time-varying disturbance is quantitatively analyzed in Sect. 3.2. Variable universe 
adaptive fuzzy controller is restated in Sect. 3.3. The composite controller is introduced in Sect. 3.4.

\subsection{Sliding mode control}

Under the view of traditional sliding mode control, the control system (5) can be stabilized with the sliding mode surface as

$s=e_{w}+c_{3} e_{z}+c_{2} e_{y}+c_{1} e_{x}$

where the coefficients $c_{1}, c_{2}, c_{3}$ satisfy the Hurwitz polynomial $p^{3}+c_{3} p^{2}+c_{2} p+c_{1}$, $p$ is Laplace operator. By changing the first term of Eq. (6), we can get a sliding mode surface with nonlinear term, i.e.

$s=e_{w}^{p / q}+c_{3} e_{z}+c_{2} e_{y}+c_{1} e_{x}$

where $q<p<2 q, p, q$ are prime numbers, the coefficients $c_{1}, c_{2}, c_{3}$ is to be designed such that the sliding surface (7) is stable when $s=0$.

Differentiating Eq. (7), we get

$$
\begin{aligned}
\dot{s} & =\frac{p}{q} e_{w}^{p / q-1} \dot{e}_{w}+c_{3} e_{w}+c_{2} e_{z}+c_{1} e_{y} \\
& =\frac{p}{q} e_{w}^{p / q-1}\left(\dot{e}_{w}+c_{3} \frac{q}{p} e_{w}^{2-p / q}+c_{2} \frac{q}{p} e_{w}^{1-p / q} e_{z}+c_{1} \frac{q}{p} e_{w}^{1-p / q} e_{y}\right)
\end{aligned}
$$

Considering the error dynamic system (5), ignoring the external disturbances, the equivalent control term is solved by setting $\dot{s}=0$

$u_{e q}=-f_{r}+\alpha f_{d}-\frac{c_{3} q}{p} e_{w}^{2-p / q}-\frac{c_{2} q}{p} e_{w}^{1-p / q} e_{z}-\frac{c_{1} q}{p} e_{w}^{1-p / q} e_{y}$

The switching control term is

$u_{e r l}=-M \operatorname{sign}(s)-\gamma s$

Then the comprehensive controller is designed as

$$
\begin{aligned}
u= & u_{e q}+u_{e r l} \\
= & -f_{r}+\alpha f_{d}-\frac{c_{3} q}{p} e_{w}^{2-p / q}-\frac{c_{2} q}{p} e_{w}^{1-p / q} e_{z}-\frac{c_{1} q}{p} e_{w}^{1-p / q} e_{y} \\
& -M \operatorname{sign}(s)-\gamma s
\end{aligned}
$$

As is well known, the main cause of chattering is the existence of switching gain $M$. Switching term is used to overcome the disturbances and uncertainty. It is a good idea by using disturbance observer to compensate the disturbance.

\subsection{Observer of slow time-varying disturbance}

In this section, we give the disturbance observer based on Kawamura's work [28] and analyze the observation error quantitatively.

For $d_{1}(t)$ of system (2), we design the disturbance observer as

$\left\{\begin{array}{l}\dot{\hat{d}}_{1}=-k_{1}\left(\hat{w}_{1}-w_{1}\right), \\ \dot{\hat{w}}_{1}=f_{d}+\hat{d}_{1}-k_{2}\left(\hat{w}_{1}-w_{1}\right),\end{array}\right.$

where $\hat{d}_{1}$ is the estimation of $d_{1}, \hat{w}_{1}$ is the estimation of $w_{1}$, $k_{1}>0, k_{2}>0$.

For $d_{2}(t)$ of system (3), we design the disturbance observer as follows

$\left\{\begin{array}{l}\dot{\hat{d}}_{2}=-k_{3}\left(\hat{w}_{2}-w_{2}\right), \\ \dot{\hat{w}}_{2}=f_{r}+\hat{d}_{2}-k_{4}\left(\hat{w}_{2}-w_{2}\right)+u,\end{array}\right.$

where $\hat{d}_{2}$ is the estimation of $d_{2}, \hat{w}_{2}$ is the estimation of $w_{2}$, $k_{3}>0, k_{4}>0$.

We define observation errors $\tilde{d}_{i}=\hat{d}_{i}-d_{i}, \tilde{w}_{i}=\hat{w}_{i}-w_{i},(i=1,2)$, now we calculate them quantificationally. From system (2) and system (12), we have

$\left\{\begin{array}{l}\dot{\tilde{d}}_{1}=-k_{1} \tilde{w}_{1}-\dot{d}_{1} \\ \dot{\tilde{w}}_{1}=\tilde{d}_{1}-k_{2} \tilde{w}_{1}\end{array}\right.$

From system (3) and system (13), we have

$\left\{\begin{array}{l}\dot{\tilde{d}}_{2}=-k_{3} \tilde{w}_{2}-\dot{d}_{2} \\ \dot{\tilde{w}}_{2}=\tilde{d}_{2}-k_{4} \tilde{w}_{2}\end{array}\right.$

By using vector notation, we define

$E_{1}=\left(\begin{array}{l}\tilde{d}_{1} \\ \tilde{w}_{1}\end{array}\right), A=\left(\begin{array}{ll}0 & -k_{1} \\ 1 & -k_{2}\end{array}\right), B=\left(\begin{array}{c}-1 \\ 0\end{array}\right)$

and rewrite the system (14) as one 2-dimensional firstorder vector differential equation

$\dot{E}_{1}=A E_{1}+B \dot{d}_{1}$

Now we diagonalize the system matrix $A$. The eigenvalues of $A$ are $\lambda_{1}=\frac{-k_{2}+\sqrt{k_{2}^{2}-4 k_{1}}}{2}$ and $\lambda_{2}=\frac{-k_{2}-\sqrt{k_{2}^{2}-4 k_{1}}}{2}$.

If we define 


$$
\begin{aligned}
D & =\left(\begin{array}{cc}
\frac{-k_{2}+\sqrt{k_{2}^{2}-4 k_{1}}}{2} & 0 \\
0 & \frac{-k_{2}-\sqrt{k_{2}^{2}-4 k_{1}}}{2}
\end{array}\right), \\
P & =\left(\begin{array}{cc}
\frac{k_{2}+\sqrt{k_{2}^{2}-4 k_{1}}}{2} & \left.\frac{k_{2}-\sqrt{k_{2}^{2}-4 k_{1}}}{2}\right), \\
1 & 1
\end{array}\right) \\
P^{-1} & =\frac{1}{\sqrt{k_{2}^{2}-4 k_{1}}}\left(\begin{array}{cc}
1 & \frac{-k_{2}+\sqrt{k_{2}^{2}-4 k_{1}}}{2} \\
-1 & \frac{k_{2}+\sqrt{k_{2}^{2}-4 k_{1}}}{2}
\end{array}\right),
\end{aligned}
$$

then

$P^{-1} A P=D, A=P D P^{-1}$

Defining $E_{1}^{*}=P^{-1} E_{1}$, then system (16) change to

$\dot{E}_{1}^{*}=D E_{1}^{*}+P^{-1} B \dot{d}_{1}$.

For $D$ is diagonalizable, from linear system theory, we know the solution of system (18) is

$E_{1}^{*}=e^{D t} E_{1}^{*}\left(t_{0}\right)+\int_{0}^{t} e^{D(t-\tau)} P^{-1} B \dot{d}_{1}(\tau) \mathrm{d} \tau$

Because $\lambda_{1}=\frac{-k_{2}+\sqrt{k_{2}^{2}-4 k_{1}}}{2}, \lambda_{2}=\frac{-k_{2}-\sqrt{k_{2}^{2}-4 k_{1}}}{2}$, then

$$
\begin{aligned}
E_{1}^{*}= & \left(\begin{array}{cc}
e^{\lambda_{1} t} & 0 \\
0 & e^{\lambda_{2} t}
\end{array}\right) E_{1}^{*}\left(t_{0}\right) \\
& +\frac{1}{\sqrt{k_{2}^{2}-4 k_{1}}}\left(\begin{array}{cc}
e^{\lambda_{1} t} & 0 \\
0 & e^{\lambda_{2} t}
\end{array}\right) \int_{0}^{t}\left(\begin{array}{c}
-e^{-\lambda_{1} \tau} \\
e^{-\lambda_{2} \tau}
\end{array}\right) \dot{d}_{1}(\tau) \mathrm{d} \tau
\end{aligned}
$$

If $\dot{d}_{1}(t)=0, E_{1}^{*} \rightarrow 0$ as $t \rightarrow \infty$.

Suppose $0<\left|\dot{d}_{1}(t)\right| \leqslant N_{1}$, we have

$\left|e^{\lambda_{1} t} \int_{0}^{t}-e^{-\lambda_{1} \tau} \dot{d}_{1}(\tau) \mathrm{d} \tau\right| \leqslant N_{1} e^{\lambda_{1} t} \int_{0}^{t} e^{-\lambda_{1} \tau} \mathrm{d} \tau=\frac{N_{1}}{\lambda_{1}}\left(e^{\lambda_{1} t}-1\right)$

$\left|e^{\lambda_{2} t} \int_{0}^{t} e^{-\lambda_{2} \tau} \dot{d}_{1}(\tau) \mathrm{d} \tau\right| \leqslant N_{1} e^{\lambda_{2} t} \int_{0}^{t} e^{-\lambda_{2} \tau} \mathrm{d} \tau=\frac{N_{1}}{\lambda_{2}}\left(e^{\lambda_{2} t}-1\right)$

From linear system theory, we know $E_{1}^{*} \leqslant \frac{-N_{1}}{\sqrt{k_{2}^{2}-4 k_{1}}}\left(\begin{array}{c}\frac{1}{\lambda_{1}} \\ \frac{1}{\lambda_{2}}\end{array}\right)$ as $t \rightarrow \infty$, i.e.

$E_{1} \leqslant \frac{N_{1}}{2 k_{1} \sqrt{k_{2}^{2}-4 k_{1}}}\left(\begin{array}{c}k_{2}^{2}-2 k_{1} \\ 2 k_{2}\end{array}\right)$

What we're interested in is $\tilde{d}_{1}(t)$. Now we know

$$
\begin{gathered}
\left|\tilde{d}_{1}(t)\right| \leqslant \frac{N_{1}\left(k_{2}^{2}-2 k_{1}\right)}{2 k_{1} \sqrt{k_{2}^{2}-4 k_{1}}}=\frac{N_{1}\left(\left(\frac{k_{2}}{k_{1}}\right)^{2}-\frac{2}{k_{1}}\right)}{2 \sqrt{\left(\frac{k_{2}}{k_{1}}\right)^{2}-\frac{4}{k_{1}}}} \sim \frac{k_{2} N_{1}}{2 k_{1}} \\
\left(k_{1} \gg k_{2}>1\right)
\end{gathered}
$$

From (22), we know that if $k_{1} \gg k_{2}$, observer error is very small, that is to say, the disturbance observer works very well.

Example 1 If $d_{1}=\sin t$, then $\dot{d}_{1}(t)=$ cost, we have

$$
\begin{aligned}
E_{11}^{*}= & e^{\lambda_{1} t} E_{11}^{*}\left(t_{0}\right)+\frac{1}{\left(1+\lambda_{1}^{2}\right) \sqrt{k_{2}^{2}-4 k_{1}}}\left(-\sin t+\lambda_{1} \cos t-\lambda_{1} e^{\lambda_{1} t}\right) \\
= & \left(E_{11}^{*}\left(t_{0}\right)-\frac{\lambda_{1}}{\left(1+\lambda_{1}^{2}\right) \sqrt{k_{2}^{2}-4 k_{1}}}\right) e^{\lambda_{1} t} \\
& +\frac{1}{\sqrt{1+\lambda_{1}^{2}} \sqrt{k_{2}^{2}-4 k_{1}}}\left(\frac{-1}{\sqrt{1+\lambda_{1}^{2}}} \sin t+\frac{\lambda_{1}}{\sqrt{1+\lambda_{1}^{2}}} \cos t\right) \\
= & \left(E_{11}^{*}\left(t_{0}\right)-\frac{\lambda_{1}}{\left(1+\lambda_{1}^{2}\right) \sqrt{k_{2}^{2}-4 k_{1}}}\right) e^{\lambda_{1} t} \\
& +\frac{1}{\sqrt{1+\lambda_{1}^{2}} \sqrt{k_{2}^{2}-4 k_{1}}} \sin \left(t+\beta_{1}\right) \\
E_{12}^{*}= & e^{\lambda_{2} t} E_{12}^{*}\left(t_{0}\right)+\frac{1}{\left(1+\lambda_{2}^{2}\right) \sqrt{k_{2}^{2}-4 k_{1}}}\left(\sin t-\lambda_{2} \cos t-\lambda_{2} e^{\lambda_{2} t}\right) \\
= & \left(E_{12}^{*}\left(t_{0}\right)-\frac{\lambda_{2}}{\left(1+\lambda_{2}^{2}\right) \sqrt{k_{2}^{2}-4 k_{1}}}\right) e^{\lambda_{2} t} \\
& +\frac{1}{\sqrt{1+\lambda_{2}^{2}} \sqrt{k_{2}^{2}-4 k_{1}}}\left(\frac{1}{\sqrt{1+\lambda_{2}^{2}}} \sin t-\frac{\lambda_{2}}{\sqrt{1+\lambda_{2}^{2}}} \cos t\right) \\
= & \left(E_{12}^{*}\left(t_{0}\right)-\frac{\lambda_{2}}{\left(1+\lambda_{2}^{2}\right) \sqrt{k_{2}^{2}-4 k_{1}}}\right) e^{\lambda_{2} t} \\
& +\frac{1}{\sqrt{1+\lambda_{2}^{2}} \sqrt{k_{2}^{2}-4 k_{1}}} \sin \left(t+\beta_{2}\right)
\end{aligned}
$$

Based on linear system theory, we know $E_{1}^{*} \rightarrow\left(\begin{array}{c}\frac{1}{\sqrt{1+\lambda_{1}^{2}} \sqrt{k_{2}^{2}-4 k_{1}}} \sin \left(t+\beta_{1}\right) \\ \frac{1}{\sqrt{1+\lambda_{2}^{2}} \sqrt{k_{2}^{2}-4 k_{1}}} \sin \left(t+\beta_{2}\right)\end{array}\right)$.

From $E_{1}=P E_{1}^{*}$, we have

$E_{1} \rightarrow\left(\begin{array}{c}\frac{-\sqrt{\left(1+k_{2}^{2}-k_{1}\right)^{2}+k_{1}^{2} k_{2}^{2}}}{1+k_{1}^{2}+k_{2}^{2}-2 k_{1}} \sin \left(t+\beta_{1}\right) \\ \frac{1}{\sqrt{\left(1-k_{1}\right)^{2}+k_{2}^{2}}} \sin \left(t+\beta_{2}\right)\end{array}\right)$ 
Note 1: From (23), we know that the period of the observer error is the same as the period of the disturbance, the observer error boundary of $\tilde{d}_{1}$ is $\frac{\sqrt{\left(1+k_{2}^{2}-k_{1}\right)^{2}+k_{1}^{2} k_{2}^{2}}}{1+k_{1}^{2}+k_{2}^{2}-2 k_{1}}$ and the observer error boundary of $\tilde{\omega}_{1}$ is $\frac{1}{\sqrt{\left(1-k_{1}\right)^{2}+k_{2}^{2}}}$.

The changes of observer error boundary with parameters evolving are shown as Table 1.

Table 1 further illustrates that as long as appropriate parameters $k_{1}$ and $k_{2}$ are selected, the observer can achieve better compensation. It is also demonstrated that the observation error is persistent as long as the derivative of the disturbance is not zero. Once again, it is proved that the observation error is related to the parameter proportion.

We also get the similar conclusions about the observer error system (15).

\subsection{Variable universe adaptive fuzzy controller}

From the discussion of the above subsection, we know the switching gain in sliding mode control can be significantly reduced by using the disturbance observer method. At the same time, as long as the derivative is not equal to zero, the observation error is persisting steadily existing. And if the disturbances don't have derivatives (inevitable, white noise, for instance), the observer cannot be employed to compensate them. To further reduce chattering is still a problem. Because the fuzzy system can estimate the switching gain effectively according to the reaching condition, this action can reduce chattering. Usually the control precision in a

Table 1 Observer errors with parameters $k 1, k 2$ changing

\begin{tabular}{lrrll}
\hline No. & \multicolumn{1}{l}{$k_{1}$} & $k_{2}$ & Boundary of $\tilde{d}_{1}$ & Boundary of $\tilde{\omega}_{1}$ \\
\hline 1 & 1000 & 50 & 0.05 & $9.9975 \times 10^{-4}$ \\
2 & 1000 & 100 & 0.0996 & $9.9602 \times 10^{-4}$ \\
3 & 1000 & 150 & 0.1485 & $9.8990 \times 10^{-4}$ \\
4 & 1000 & 200 & 0.1963 & $9.8152 \times 10^{-4}$ \\
5 & 1000 & 250 & 0.2428 & $9.7106 \times 10^{-4}$ \\
6 & 2000 & 100 & 0.05 & $4.9963 \times 10^{-4}$ \\
7 & 3000 & 100 & 0.0333 & $3.3326 \times 10^{-4}$ \\
8 & 4000 & 100 & 0.0250 & $2.4998 \times 10^{-4}$ \\
9 & 5000 & 100 & 0.0200 & $2.000 \times 10^{-4}$ \\
10 & 6000 & 100 & 0.0167 & $1.6667 \times 10^{-4}$ \\
11 & 7000 & 100 & 0.0143 & $1.4286 \times 10^{-4}$ \\
12 & 8000 & 100 & 0.0125 & $1.2501 \times 10^{-4}$ \\
13 & 9000 & 100 & 0.0111 & $1.1112 \times 10^{-4}$ \\
14 & 10,000 & 100 & 0.0100 & $1.0000 \times 10^{-4}$ \\
15 & 3000 & 150 & 0.05 & $3.3303 \times 10^{-4}$ \\
\hline
\end{tabular}

fuzzy system can be improved by increasing the amount of fuzzy rules. Meanwhile, this can lead to a large number of computations and even an "rules explosion." VUAFC method improves the control precision by on-line contraction-expansion of variable universes without increasing the fuzzy rules. Variable universe adaptive fuzzy control method can establish high control precision with a few fuzzy rules.

Variable universe adaptive fuzzy controller was first proposed by Li $[33,34]$. Because this is a high precision controller, many scholars have studied it [35-38]. Suppose $X_{i}=\left[-E_{i}, E_{i}\right](i=1,2, \ldots, m)$ be the universe of input variable $x_{i}(i=1,2, \ldots, m), Y=[-U, U]$ be the universe of the output variable $y .\left\{A_{i j}\right\}\left(1 \leqslant j \leqslant q_{i}\right)$ stand for a fuzzy partition on $X_{i}$ and $B_{l}(1 \leqslant l \leqslant h)$ stand for a fuzzy partition on $Y$. Suppose the fuzzy inference rules is as:

If $x_{1}$ is $A_{1 j_{1}}, x_{2}$ is $A_{2 j_{2}}, \ldots x_{m}$ is $A_{m j_{m}}$ then $y$ is $B_{1}$.

Suppose $x_{i j_{i}}$ is the peakpoint of $A_{i j_{i}} y_{l}$ is the peakpoint of $B_{l}$. The output of canonical fuzzy controller is

$y=\sum_{j_{1}=1}^{q_{1}} \ldots \sum_{j_{m}=1}^{q_{m}} \prod_{i=1}^{m} A_{i j_{i}}\left(x_{i}\right) y_{1}$.

By introducing scale factors $\alpha_{i}$ and $\beta$, the universe discourse is changed with the changing variables $x_{i}$ and $y_{1}$ i.e. $X_{i}\left(x_{i}\right)=\left[-\alpha_{i}\left(x_{i}\right) E_{i}, \alpha_{i}\left(x_{i}\right) E_{i}\right], Y(y)=[-\beta(y) U, \beta(y) U]$. The fuzzy output of the variable universe fuzzy controller is as

$y=\beta \sum_{j_{1}=1}^{q_{1}} \cdots \sum_{j_{m}=1}^{q_{m}} \prod_{i=1}^{m} A_{i j_{i}}\left(\frac{x_{i}}{\alpha\left(x_{i}\right)}\right) y_{l}$.

The parameter $\beta$ can be adjusted by optimization.

\subsection{The design of the controller}

Based on (25), we firstly design the fuzzy system, the input of the fuzzy system is selected as sliding mode surface $s(t)$. Let the output of the fuzzy system is $\mu$. The universes of discourse of $s(t)$ and $\mu$ are $s_{0} \mid \times[-1,1]$ and $[-1,1]$, respectively.

Suppose the width of fuzzy layer is $2 \delta$, the initial membership function of $s(t)$ is:

$$
\begin{aligned}
& N B(s)=\min \left(1, \max \left(-\frac{s}{\delta}, 0\right)\right), \\
& Z O(s)=\max \left(0, \min \left(\frac{s+\delta}{\delta},-\frac{s-\delta}{\delta}\right)\right), \\
& P B(s)=\min \left(1, \max \left(\frac{s}{\delta}, 0\right)\right)
\end{aligned}
$$

The plot of initial membership function is depicted by Fig. 1.

The initial membership function of $\mu$ is selected as: 


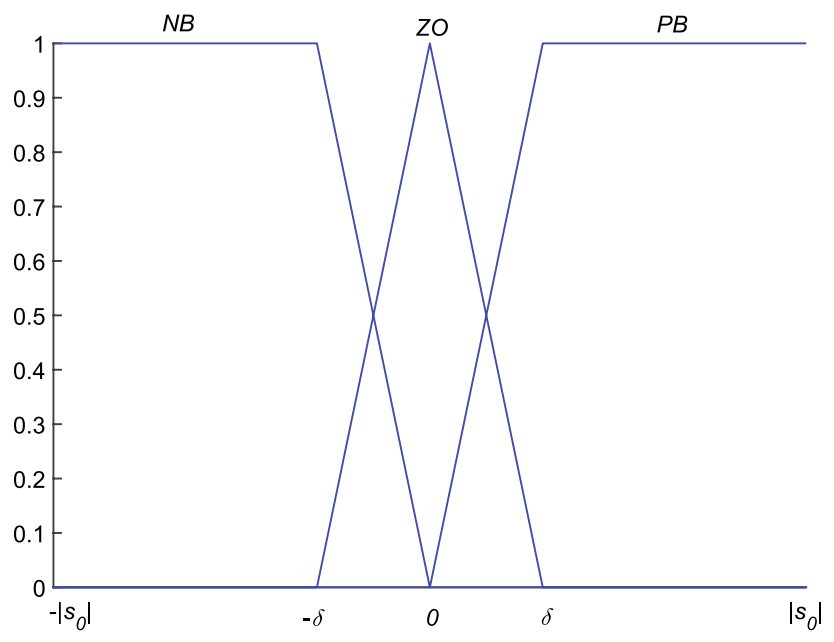

Fig. 1 The initial membership function of input $s(t)$

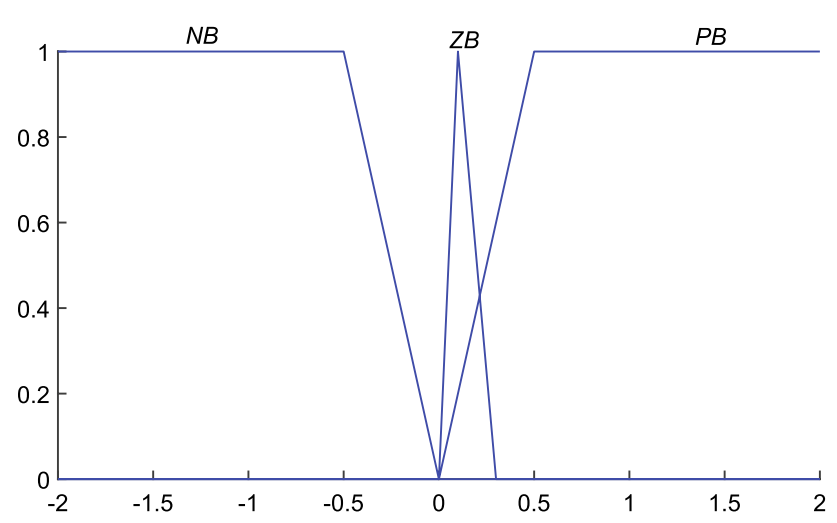

Fig. 2 The initial membership function of output $\mu$

$$
\begin{aligned}
& N B(\mu)=\min \left(1, \max \left(-\frac{\mu}{0.5}, 0\right)\right), \\
& Z O(\mu)=\max \left(0, \min \left(\frac{\mu}{0.1},-\frac{\mu-0.3}{0.2}\right)\right), \\
& P B(\mu)=\min \left(1, \max \left(\frac{\mu}{0.5}, 0\right)\right)
\end{aligned}
$$

The plot of initial membership function is depicted by Fig. 2.

The natural initial fuzzy rules are:

$R^{(1)}$ : If $s(t)$ is $N B$ then $\mu$ is $P B$

$R^{(2)}$ : If $s(t)$ is $Z O$ then $\mu$ is $Z O$

$R^{(3)}$ : If $s(t)$ is $P B$ then $\mu$ is $P B$

The contraction-expansion factor is as follows $\alpha(s)=1-\lambda_{1} \exp \left(-\lambda_{2} s^{2}\right), \quad \lambda_{1} \in(0,1), \lambda_{2}>0$

From (25), we know the structure of fuzzy system is

$$
\begin{aligned}
\mu= & \left(p_{1} \int_{0}^{t} s \mathrm{~d} \tau+\beta(0)\right)\left(0.5 N B\left(\frac{s}{\alpha(s)}\right)\right. \\
& \left.+0.1 Z O\left(\frac{s}{\alpha(s)}\right)+0.5 P B\left(\frac{s}{\alpha(s)}\right)\right)
\end{aligned}
$$

We add a coefficient $\mu$ to the switch term, the control law is designed as

Theorem 1 With the disturbance observers (12) and (13), projective synchronization of system (2) and system (3) up to scaling factor $\alpha$ can be realized under the following controller

$$
\begin{aligned}
u= & -f_{r}+\alpha f_{d}-\hat{d}_{2}(t)+\alpha \hat{d}_{1}(t)-\frac{c_{3} q}{p} e_{w}^{2-p / q} \\
& -\frac{c_{2} q}{p} e_{w}^{1-p / q} e_{z}-\frac{c_{1} q}{p} e_{w}^{1-p / q} e_{y} \\
& -\mu M_{2} \operatorname{sign}(s)-\gamma_{2} s
\end{aligned}
$$

where $M_{2} \geqslant\left|D_{1}\right|+\left|\alpha D_{2}\right|+\left|N_{1}\right|+\left|\alpha N_{2}\right|, \mu$ is the output of variable universe adaptive fuzzy controller. The equivalent control part $u_{e q}=-f_{r}+\alpha f_{d}-\hat{d}_{2}(t)+\alpha \hat{d}_{1}(t)-\frac{c_{3} q}{p} e_{w}^{2-p / q}$ $-\frac{c_{2} q}{p} e_{w}^{1-p / q} e_{z}-\frac{c_{1} q}{p} e_{w}^{1-p / q} e_{y}$ and the reaching control part $u_{e r l}=-\mu M_{2} \operatorname{sign}(s)-\gamma_{2} s$.

Note 2: Obviously when $\mu=1, u=u_{\text {eq }}+u_{\text {erl }}$. When $\mu \neq 1$, the control law is fuzzified. We select $\left|s_{0}\right| \times[-1,1]$ as universe of discourse of $s(t)$ avoiding overflow of input.

\section{Numerical simulation}

We select the drive and response system given by [39]. The drive system is

$$
\left\{\begin{array}{l}
\dot{x}_{1}=y_{1}, \\
\dot{y}_{1}=z_{1} \\
\dot{z}_{1}=w_{1} \\
\dot{w}_{1}=-2 x_{1}^{3}-y_{1}^{3}+\left(3 x_{1}-4 z_{1}\right) z_{1}^{2}-0.1 w_{1}+d_{1}(t)+n_{1}(t) .
\end{array}\right.
$$

where $d_{1}(t)=3 \sin (3 t)$ is the low time-varying disturbance and $n_{1}(t)=0.1$ rand is uniform white noise.

The response system is 


$$
\left\{\begin{array}{l}
\dot{x}_{2}=y_{2} \\
\dot{y}_{2}=z_{2} \\
\dot{z}_{2}=w_{2} \\
\dot{w}_{2}=-2 x_{2}^{3}-y_{2}^{3}+\left(3 x_{2}-4 z_{2}\right) z_{2}^{2}-0.1 w_{2}+d_{2}(t)+n_{2}(t) .
\end{array}\right.
$$

where $d_{2}(t)=\sin (0.2 t)+\cos (t)$ is the low time-varying disturbance and $n_{2}(t)=0.2 \mathrm{rand}(t)$ is uniform white noise.

We select the scaling factor $\alpha=1 / 2$, and the initial values of drive system and response system are $(3,3,3,-3)^{T}$ and $(-1,-1,-1,-1)^{T}$, respectively.

The sliding mode surface is designed as

$s=e_{w}^{\frac{5}{3}}+3 e_{z}+3 e_{y}+e_{x}$

Let the parameters $k_{1}=1000, k_{2}=200 \mathrm{in} \mathrm{Eq.} \mathrm{(12).} \mathrm{Let} \mathrm{the}$ parameters $k_{3}=1000, k_{4}=200 \mathrm{in} \mathrm{Eq.} \mathrm{(13).} \mathrm{Let} \mathrm{the} \mathrm{param-}$ eters $M_{2}=10, \gamma_{2}=50$.

\subsection{Sliding mode control with disturbance observer}

Regardless of fuzzy system, that is to say, let $\mu=1$ in Eq. (29).

The synchronization error is depicted as Fig. 3. The evolution of sliding mode surface is depicted as Fig. 4.

The control $u$ is depicted as Fig. 5. For the sake of clarity, the control $u$ is enlarged as Fig. 6 . The chattering phenomenon is very obvious.

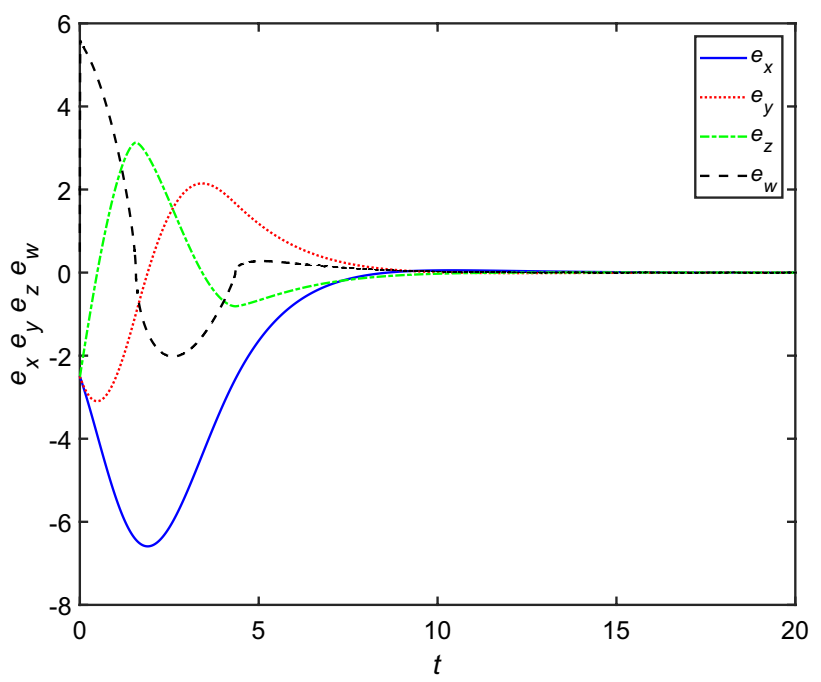

Fig. 3 The synchronization errors

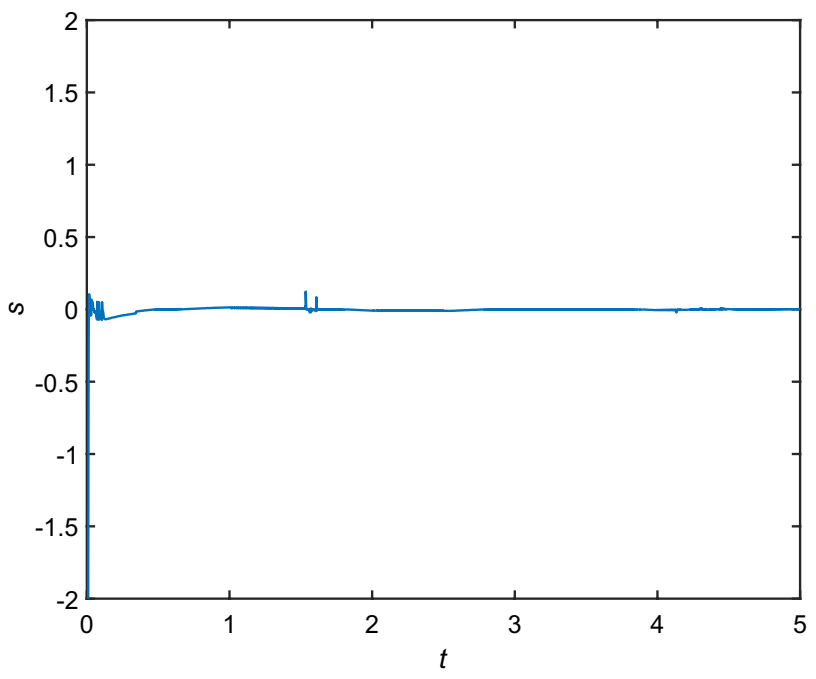

Fig. 4 The evolution of sliding mode surface $s$

The phase diagrams of projective synchronization are depicted as Figs. 7 and 8.

Figure 3 illustrates the projective synchronization be achieved with $\mu=1$ of controller (28). Figures 5 and 6 illustrate the presence of chattering.

\subsection{Sliding mode control with disturbance observer and variable universe adaptive fuzzy controller}

Suppose the parameter $\delta=1$ of Eq. (26), $\lambda_{1}=0.9, \lambda_{2}=10$ in Eq. (27), with the variable universe adaptive fuzzy

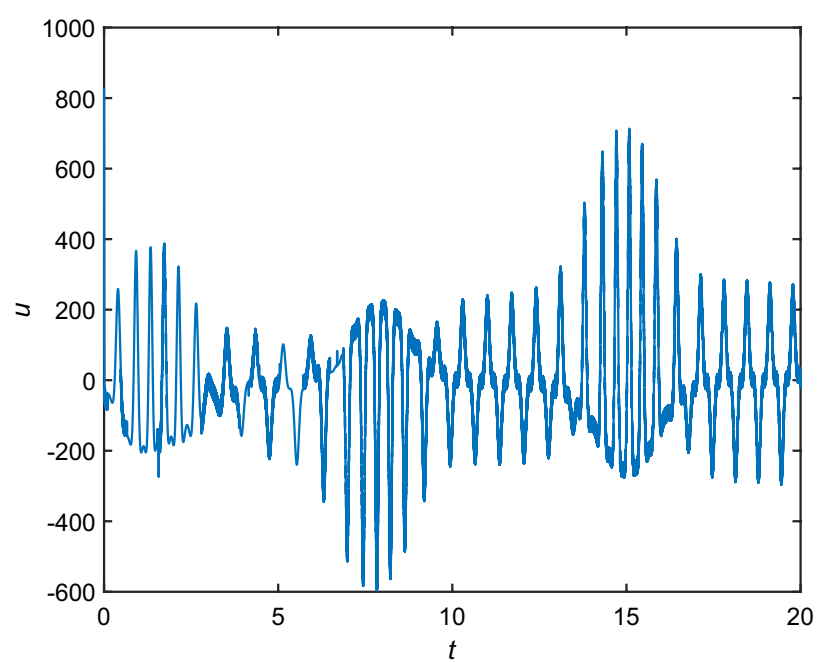

Fig. 5 The evolution of control $u$ 


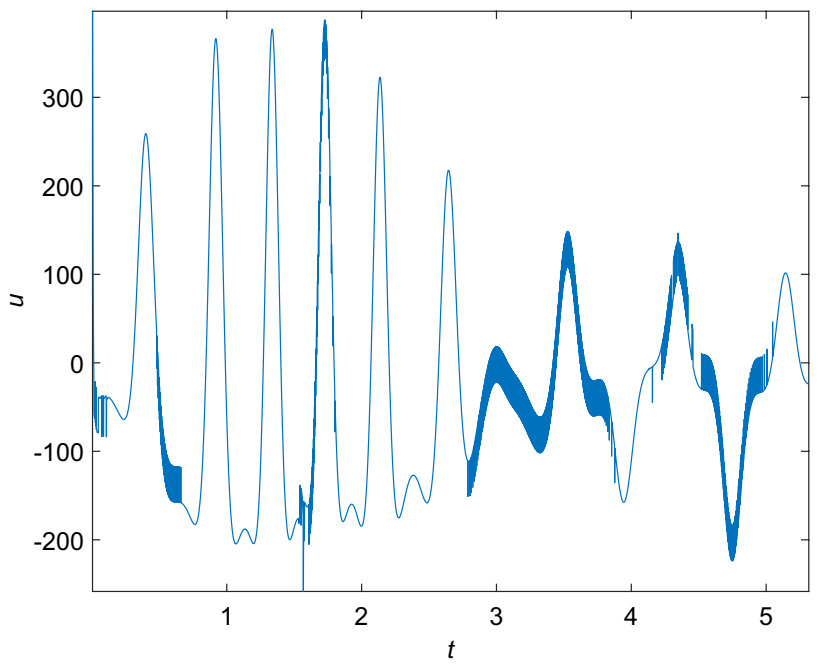

Fig. 6 The enlargement of control $u$

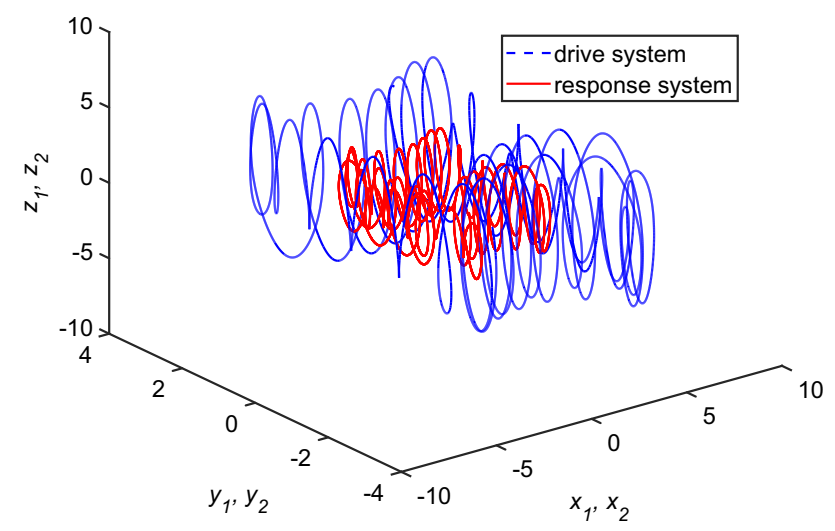

Fig. 7 The phase diagram on the $x_{1}-y_{1}-z_{1}\left(x_{2}-y_{2}-z_{2}\right)$ space

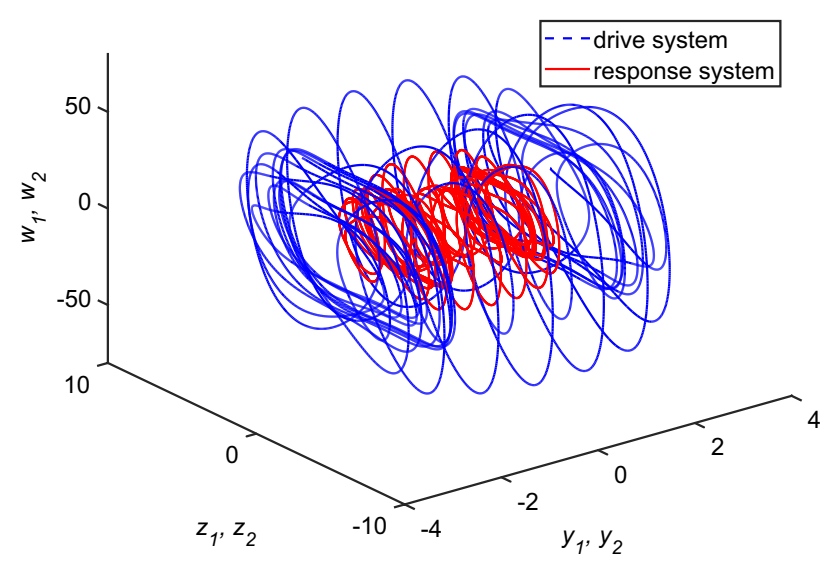

Fig. 8 The phase diagram on the $y_{1}-z_{1}-w_{1}\left(y_{2}-z_{2}-w_{2}\right)$ space SN Applied Sciences

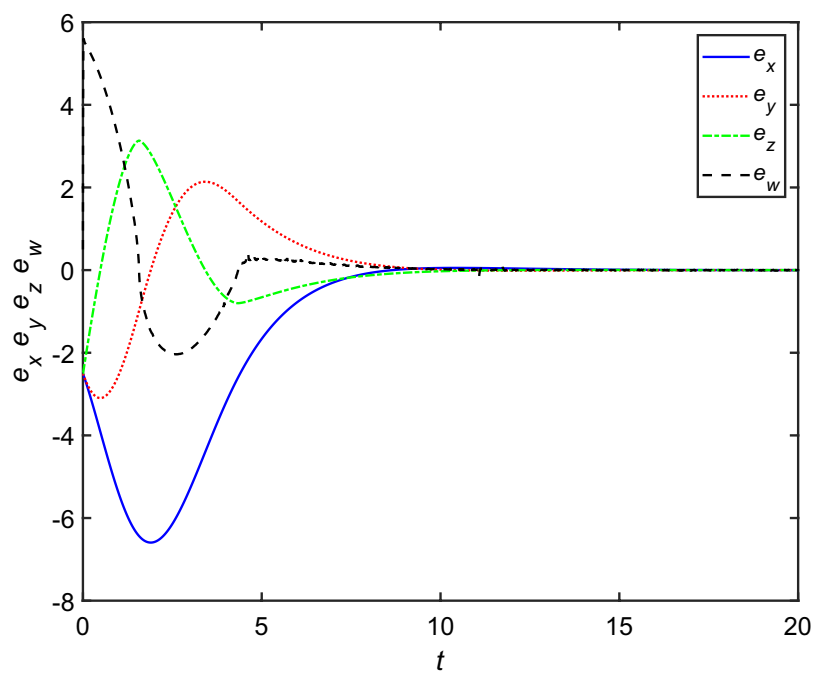

Fig. 9 The evolution of the synchronization errors

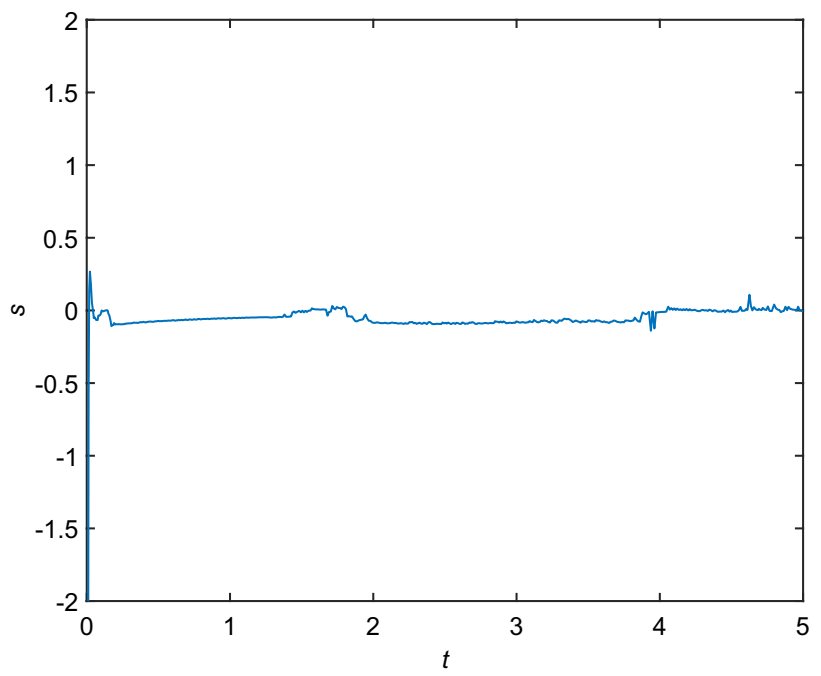

Fig. 10 The evolution of sliding mode surface $s$

controller (28), the simulation results of composite controller (29) are as follows.

The synchronization error is depicted as Fig. 9. The evolution of sliding mode surface $s$ is depicted as Fig. 10.

The control $u$ is depicted as Fig. 11. For the sake of clarity, the control $u$ is enlarged as Fig. 12.

The phase diagrams of projective synchronization are depicted as Figs. 13 and 14.

From Fig. 9, we know that the projective synchronization is achieved with controller (29). By comparing Figs. 5, 6, 11 and 12, we find, by using VUAFC, chattering phenomenon is eliminated. Meanwhile, from Figs. (4) and (10), we find that $s$ cannot stay at zero. 


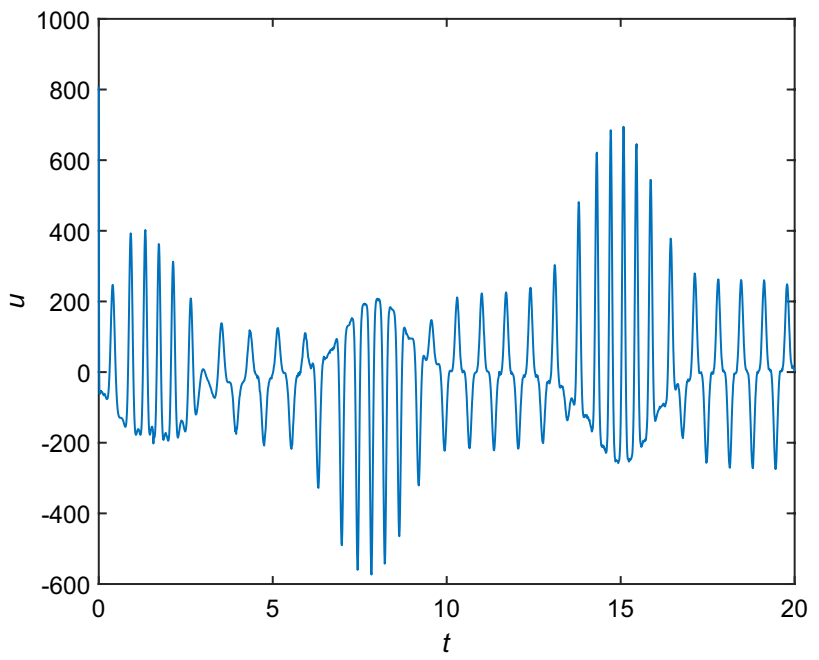

Fig. 11 The control $u$

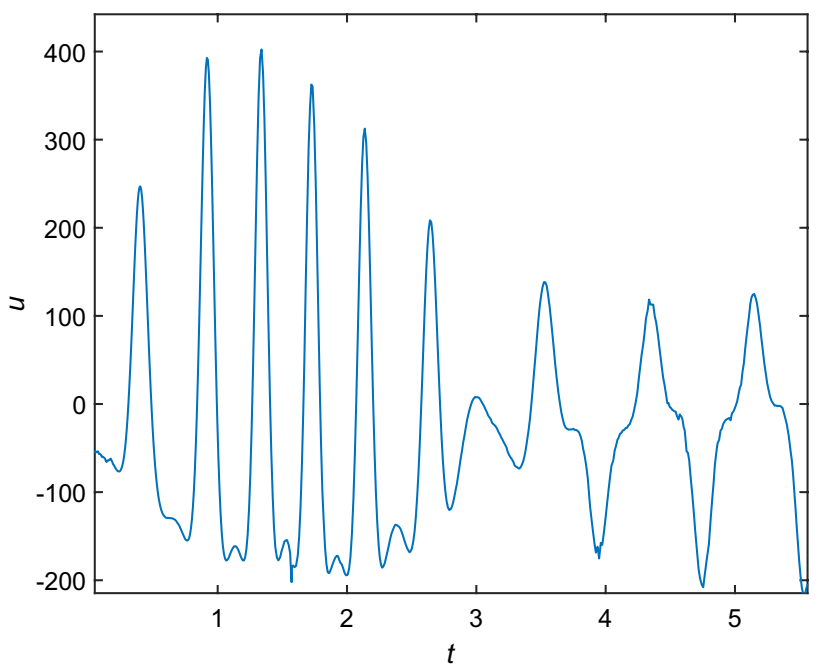

Fig. 12 The enlargement of the control $u$

\section{Conclusion}

In this paper, we have discussed fuzzy sliding mode projective synchronization of hyperjerk system with disturbances. Disturbance observer has been developed for the low time-varying disturbance. By quantitative analysis, given appropriate observer parameters, the disturbance can be compensated by the observer. For the chattering caused by the white noise and the persistent observer error, we have used VUAFC method to smooth it. VUAFSMPS method which has been designed is built on sliding mode control, disturbance observer and variable universe adaptive fuzzy control. Simulation results have shown that

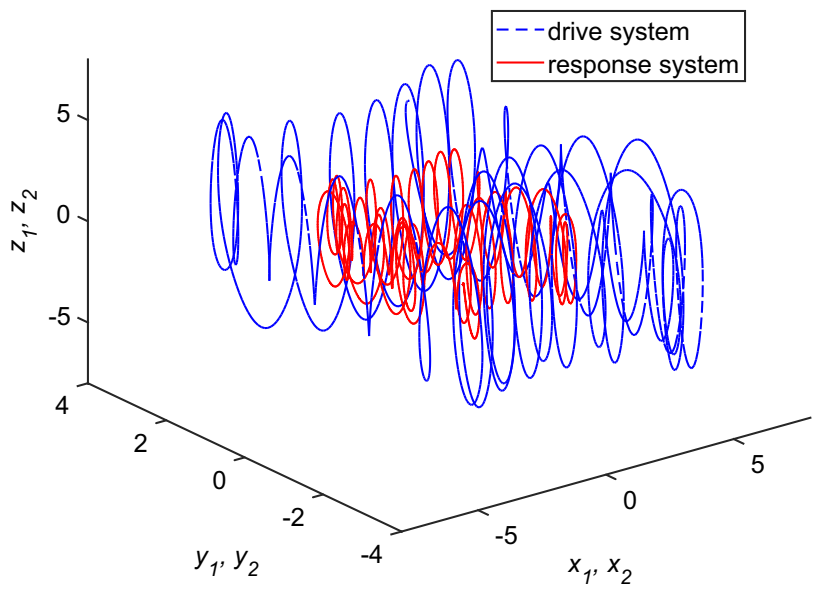

Fig. 13 The phase diagram on the $x_{1}-y_{1}-z 1\left(x_{2}-y_{2}-z_{2}\right)$ space

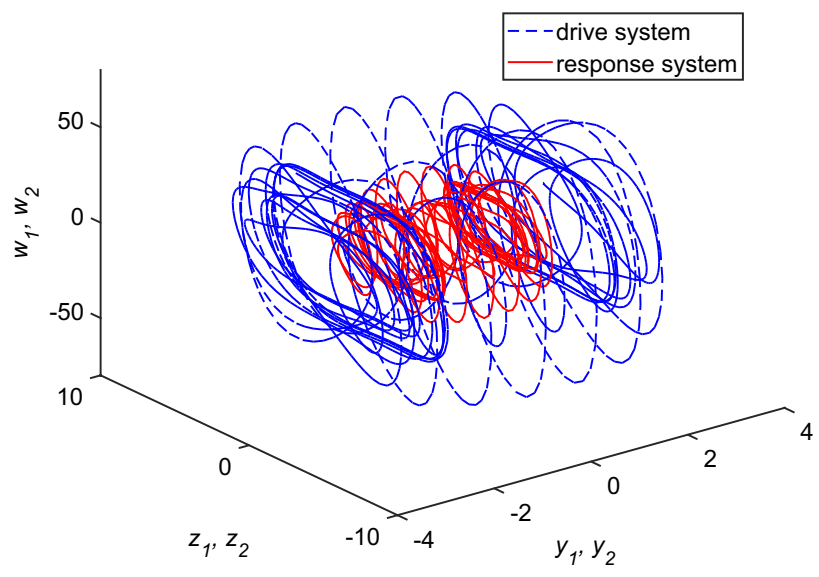

Fig. 14 The phase diagram on the $y_{1}-z_{1}-w_{1}\left(y_{2}-z_{2}-w_{2}\right)$ space

this control method can reduce chattering significantly without increasing the amount of control.

Acknowledgements This work is partly supported by Foundation of Chongqing Municipal Key Laboratory of Institutions of Higher Education (Grant No. [2017]3), the Natural Science Foundation of Chongqing, China (Grant No. cstc2018jcyjA2453) and the Scientific and Technological Research Program of Chongqing Municipal Education Commission (Grant No. KJQN201801214).

Data availability The datasets generated during and/or analyzed during the current study are available from the corresponding author on reasonable request. 


\section{Declarations}

Conflict of interest The authors declare that they have no conflict of interest regarding the publication of this paper.

Open Access This article is licensed under a Creative Commons Attribution 4.0 International License, which permits use, sharing, adaptation, distribution and reproduction in any medium or format, as long as you give appropriate credit to the original author(s) and the source, provide a link to the Creative Commons licence, and indicate if changes were made. The images or other third party material in this article are included in the article's Creative Commons licence, unless indicated otherwise in a credit line to the material. If material is not included in the article's Creative Commons licence and your intended use is not permitted by statutory regulation or exceeds the permitted use, you will need to obtain permission directly from the copyright holder. To view a copy of this licence, visit http://creativecommons. org/licenses/by/4.0/.

\section{References}

1. Chlouverakis KE, Sprott JC (2006) Chaotic hyperjerk systems. Chaos Solitons Fractal 28(3):739-746

2. Schot SH (1998) Jerk: the time rate of change of acceleration. Am J Phys 46(11):1090-1094

3. Ma J, Wu X, Chu R, Zhang L (2014) Selection of multi-scroll attractors in jerk circuits and their verification using Pspice. Nonlinear Dyn 76(4):1-12

4. Vaidyanathan S, Akgul A, Kacar S, Cavusoğlu U (2018) A new 4-D chaotic hyperjerk system, its synchronization, circuit design and applications in RNG, image encryption and chaosbased steganography. Eur Phys J Plus 133(2):46

5. Vaidyanathan S, Sambas A, Afendee M, Mamat M, Sanjaya M (2018) A new hyperchaotic hyperjerk system with three nonlinear terms, its synchronization and circuit simulation. Int J Eng Technol 7(3):1585-1592

6. Vaidyanathan S, Volos C, Pham VT, Madhavan K (2015) Analysis, adaptive control and synchronization of a novel 4-D hyperchaotic hyperjerk system and its SPICE implementation. Arch Control Sci 25(1):135-158

7. Vaidyanathan S (2016) Analysis, adaptive control and synchronization of a novel 4-D hyperchaotic hyperjerk system via backstepping control method. Arch Control Sci 26(3):311-338

8. Leutcho GD, Kengne J, Kengne LK (2018) Dynamical analysis of a novel autonomous 4-D hyperjerk circuit with hyperbolic sine nonlinearity: chaos, antimonotonicity and a plethora of coexisting attractors. Chaos Solitons Fractals 107:67-87

9. Da Ltzis P, Vaidyanathan S, Pham V, Volos C, Nistazakis E, Tombras G (2018) Hyperchaotic attractor in a novel hyperjerk system with two nonlinearities. Circuits Syst Signal Process 37(2):613-635

10. Dalkiran FY, Sprott JC (2016) Simple chaotic hyperjerk system. Int J Bifurc Chaos 26(11):1-16

11. Wang X, Vaidyanathan S, Volos C, Pham V, Kapitaniak T (2017) Dynamics, circuit realization, control and synchronization of a hyperchaotic hyperjerk system with coexisting attractors. Nonlinear Dyn 1(3):1-15

12. Mainieri R, Rehacek J (1999) Projective synchronization in three-dimensional chaotic systems. Phys Rev Lett 82(15):3042-3045

13. Khan A, Nigar U (2020) Combination projective synchronization in fractional-order chaotic system with disturbance and uncertainty. Int J Appl Comput Math 6(97):1-22
14. Fu Q, Zhong S, Jiang W, Xie W (2020) Projective synchronization of fuzzy memristive neural networks with pinning impulsive control. J Frankl Inst 357(15):10387-10409

15. Ray SS (2021) Stability analysis and modified projective synchronization of fractional-order hyperchaotic dynamical systems using nonlinear controllers. Int J Mod Phys C 32(6):2150081

16. Yang S, Hu C, Yu J, Jiang H (2021) Projective synchronization in finite-time for fully quaternion-valued memristive networks with fractional-order. Chaos Solitons Fractals 147:110911

17. Song Z, Sun K (2017) Adaptive compensation control for attitude adjustment of quad-rotor unmanned aerial vehicle. Isa Trans 69:242-255

18. Singh JP, Pham VT, Hayat T, Jafari S, Alsaadi FE, Roy BK (2018) A new four-dimensional hyperjerk system with stable equilibrium point, circuit implementation, and its synchronization by using an adaptive integrator backstepping control. Chin Phys B 27(10):100501

19. Khan A, Xie W, Zhang B, Liu LW (2021) A survey of interval observers design methods and implementation for uncertain systems. J Frankl Inst 358:3077-3126

20. Khan A, Bai X, Zhang B, Yan P (2021) Interval state estimator design for Linear Parameter Varying (LPV) systems. IEEE Trans Circuits Syst II Express Briefs. https://doi.org/10.1109/TCSII.2021. 3057107

21. Fei J, Wang $H$, Fang $Y$ (2021) Novel neural network fractionalorder sliding-mode control with application to active power filter. IEEE Trans Syst Man Cybern Syst. https://doi.org/10.1109/ TSMC.2021.3071360

22. Fei J, Wang Z, Liang X, Feng Z, Xue Y (2021) Fractional sliding mode control for micro gyroscope based on multilayer recurrent fuzzy neural network. IEEE Trans Fuzzy Syst. https://doi.org/10. 1109/TFUZZ.2021.3064704

23. Fei J, Feng $Z$ (2020) Fractional-order finite-time super-twisting sliding mode control of micro gyroscope based on double-loop fuzzy neural network. IEEE Trans Syst Man Cybern Syst. https:// doi.org/10.1109/TSMC.2020.2979979

24. Gao W, Cheng M (1989) On the quality control of variable structure system. Control Decis 4(4):1-6 (in Chinese)

25. Gao W (1993) Variable structure control of nonlinear systems: a new approach. IEEE Trans Ind Electr 40:45-55

26. Hung JY, Gao W, Hung JC (1998) Variable structure control: a survey. IEEE Trans Ind Electron 40(1):2-22

27. Zak M (1988) Terminal attractors for addressable memory in neural networks. Phys Lett A 133(1-2):18-22

28. Kawamura A, Itoh H, Sakamoto K (1992) Chattering reduction of disturbance observer based sliding mode control. IEEE Trans Ind Appl 30(2):456-461

29. Lü L, Yu M, Li C, Liu S, Yan B, Chang H et al (2013) Projective synchronization of a class of complex network based on high-order sliding mode control. Nonlinear Dyn 73(1-2):411-416

30. Sariyildiz E, Oboe R, Ohnishi K (2019) Disturbance observerbased robust control and its applications: 35th anniversary overview. IEEE Trans Ind Electron PP(99):1

31. Gao Z, Guo G (2020) Fixed-time sliding mode formation control of auvs based on a disturbance observer. Acta Autom Sin Engl Ed 7(2):539-545

32. Zhang B, Guo H (2015) Universal function projective lag synchronization of chaotic systems with uncertainty by using active sliding mode and fuzzy sliding mode control. Nonlinear Dyn 81(1-2):1-13

33. Li H (1999) Adaptive fuzzy controllers based on variable universe. Sci China 42(1):10-20

34. Li H, Miao Z, Wang J (2002) Variable universe stable adaptive fuzzy control of nonlinear system. Comput Math Appl 45(3):225-240 
35. Wang J, Qiao GD, Deng B (2005) $H_{\infty}$ variable universe adaptive fuzzy control for chaotic system. Chaos Solitons Fractals 24(4):1075-1086

36. Zhou S, Wang J, Che Y, Deng B (2007) Synchronization control of Hodgkin-Huxley neurons exposed to sinusoidal electric field. In: Chinese control conference, pp 117-121

37. Zhang B, Li H, Guo H (2013) A new hyperchaotic system and the synchronization using active variable universe adaptive fuzzy controller. Math Probl Eng 2013(pt.12):1-10
38. Guo HG, Zhang BJ (2014) Observer-based variable universe adaptive fuzzy controller without additional dynamic order. Int J Autom Comput 11(4):418-425

39. Chlouverakis KE, Sprott JC (2006) Chaotic hyperjerk systems. Chaos Solitons Fractals 28(3):739-746

Publisher's Note Springer Nature remains neutral with regard to jurisdictional claims in published maps and institutional affiliations. 